\title{
The vestibular system modulates the contributions of head and torso to egocentric spatial judgements
}

\author{
Elisa R. Ferrè ${ }^{1}$ (D) Adrian J. T. Alsmith ${ }^{2}$. Patrick Haggard ${ }^{3} \cdot$ Matthew R. Longo $^{4}$
}

Received: 9 March 2020 / Accepted: 20 April 2021 / Published online: 4 June 2021

(c) The Author(s) 2021

\begin{abstract}
Egocentric representations allow us to describe the external world as experienced from an individual's bodily location. We recently developed a novel method of quantifying the weight given to different body parts in egocentric judgments (the Misalignment Paradigm). We found that both head and torso contribute to simple alter-egocentric spatial judgments. We hypothesised that artificial stimulation of the vestibular system would provide a head-related signal, which might affect the weighting given to the head in egocentric spatial judgments. Bipolar Galvanic Vestibular Stimulation (GVS) was applied during the Misalignment Paradigm. A Sham stimulation condition was also included to control for non-specific effects. Our data show that the weight given to the head was increased during left anodal and right cathodal GVS, compared to the opposite GVS polarity (right anodal and left cathodal GVS) and Sham stimulation. That is, the polarity of GVS, which preferentially activates vestibular areas in the right cerebral hemisphere, influenced the relative weightings of head and torso in egocentric spatial judgments.
\end{abstract}

Keywords Egocentric representation · Galvanic vestibular stimulation · Vestibular system · Misalignment paradigm · Multisensory integration

\section{Introduction}

When describing our surroundings we may need to use expressions such as "on my left", "on my right", etc., to facilitate the hearer's imagination of a scene, or mark an important contrast between the spatial relations of the speaker and the hearer relative to a common environment. In doing so, we are making use of a common cognitive

Communicated by Melvyn A. Goodale.

Elisa R. Ferrè

e.ferre@rhul.ac.uk

$\triangle$ Matthew R. Longo

m.longo@bbk.ac.uk

1 Department of Psychology, Royal Holloway, University of London, London, UK

2 Department of Philosophy, King's College London, London, UK

3 Institute of Cognitive Neuroscience, University College London, London, UK

4 Department of Psychological Sciences, Birkbeck, University of London, London, UK resource, the capacity for egocentric spatial representation. Egocentric representations describe the external world as experienced from an individual's location, in a manner sensitive to how the individual's body is disposed (Jeannerod and Biguer, 1987). The body is therefore considered the point of origin of egocentric representations (Bermúdez 1998, 2005). Critically, however, bodies are not points; they are extended three-dimensional objects with articulated joints and independently mobile parts. Changes in body posture potentially dissociate reference frames anchored to different body parts. Thus, different body parts may function as origins of the egocentric reference frame.

The contribution of individual body parts in influencing egocentric spatial judgments is highlighted by Peacocke's (1992) Buckingham Palace thought experiment (p. 62):

"Looking straight ahead at Buckingham Palace is one experience. It is another to look at the palace with one's face still toward it but with one's body turned toward a point on the right. In this second case, the palace is experienced as being off to one side from the direction of straight ahead, even if the view remains exactly the same as in the first case." 
This example captures the intuition that changes of body orientation can dissociate the relative spatial relations of objects to different body parts, highlighting the problem of which body part - if any — serves as the origin of egocentric representations. Both the head and torso are strong candidates for this role. On one side, the head hosts the majority of sensory organs-the eyes, ears, and the vestibular receptors-which provide a constant flow of afferent sensory information (Sherrington 1907; Avillac et al. 2005). On the other side, the torso is probably the most stable anchor for the construction of a consistent egocentric representation (Karnath et al. 1991; Serino et al. 2015), as the "great continent" of the body (Alsmith and Longo 2014).

We have recently developed a Misalignment Paradigm which isolates the respective contribution of the head and torso to egocentric spatial judgments (Alsmith et al. 2017; Longo et al. 2020). This paradigm is essentially an experimentalization of Peacocke's (1992) Buckingham Palace thought experiment, described above. We showed participants a bird's eye view of an avatar whose head was turned $45^{\circ}$ to the left or right of the torso and asked them to judge whether objects were "to the person's left" or "to the person's right" (see Fig. 1A). By measuring how these judgments change as a function of the position of the object relative to the head and torso, we determined the contributions of each body part to egocentric spatial judgments. Our results suggested that both head and torso contribute to egocentric spatial judgments, though with greater weight given to the torso in most participants. Interestingly, individual differences in the weighting of the two body parts were correlated across different spatial axes and stable over time (Longo et al. 2020).

It remains unclear, however, whether the contribution of the head and torso to egocentric spatial judgments is a static stored representation reflecting primarily semantic knowledge about body morphology, or a dynamic, continuously updated sensory representation, perhaps reflecting the saliency of afferent sensory signals. Here we investigated whether the weight given to the head and torso in the Misalignment Paradigm could be manipulated by changing the saliency of afferent sensory signals.

Visual, auditory, proprioceptive and vestibular signals could all contribute to egocentric spatial judgements (Jeannerod 1988; Blouin et al. 1996, 1998). However, the vestibular signals seem to be particularly relevant (Schilder 1935; Lhermitte 1952; Bonnier, 1905). As Sherrington (1907) noted, the vestibular system is a significant source of information about self-orientation, given that it "maintains not merely a limb in flexion or extension, but a posture of the whole animal in regard to gravitation" (p. 480). The vestibular system is a sophisticated set of sensory transducer organs that respond to motion of the head. It comprises three orthogonal semi-circular canals (anterior, posterior and horizontal) that sense rotational acceleration of the head in three-dimensional space, around the yaw, roll, and pitch axes, and two otolith organs (the utricle and saccule) that sense translational acceleration, including the orientation of the head relative to gravity. The vestibular system provides an important reference for control of the head in space (Lackner and DiZio 2005); any movement of the head generates a flow of vestibular acceleration signals which are integrated with input from other sensory modalities from vision and neck muscles. Not surprisingly, the vestibular system is highly interlinked with both visual and proprioceptive systems, with a large number of thalamic neurons responding to both vestibular, visual and proprioceptive inputs (Deecke et al. 1977; Schwarz et al. 1973). The cortical vestibular system is also strongly integrated with other sensory modalities. Neuroimaging studies have identified a widespread vestibular network in the human brain, which includes the Temporo-Parietal Junction (TPJ), posterior insula, superior temporal gyrus, Inferior Parietal Lobule (IPL), Anterior Cingulate Cortex (ACC), fronto-parietal operculum, both primary and secondary somatosensory cortices and the prefrontal cortex (Lopez et al. 2012; Zu Eulenburg et al. 2012). Functional responses suggest that the posterior parietal operculum is the core area of the human vestibular network (Eickhoff et al. 2006a, b). Critically, this area contains not only the representation of the body, but also accurate body-in-theworlds maps (Knox et al. 2006).

Vestibular signals play a role in determine the location of environmental objects in respect to the body (Clement et al. 2009) and are of central importance in structuring individuals' experience of the world in relation to themselves and others (Lopez et al. 2010; Deroualle and Lopez, 2014; Lenggenhager and Lopez 2015; Pavlidou et al. 2018). We hypothesised that artificial stimulation of the vestibular system would provide a head-related acceleration signal, which might increase the saliency of headrelated signals, and therefore affect the weighting given to the head in performance of the Misalignment Paradigm (Alsmith et al. 2017). We have used low-intensity bipolar Galvanic Vestibular Stimulation to non-invasively stimulate the vestibular receptors (Fitzpatrick and Day, 2004). An anode and cathode were placed on the left and right mastoid, or vice versa (Fig. 1B). Perilymphatic cathodal currents depolarize the trigger site and lead to excitation, whereas anodal currents hyperpolarize it resulting in inhibition (Goldberg et al. 1984). Galvanic Vestibular Stimulation causes polarity-dependent behavioural effects which are consistent with neuroimaging evidence revealing asymmetrical cortical vestibular projections in the non-dominant hemisphere of right-handed participants (Dieterich et al. 2003). We investigated whether a Galvanic Vestibular Stimulation induced bias on spatial egocentric 
judgments supports a dynamic sensory-driven egocentric representation.

\section{Methods}

\section{Participants}

Fifteen right-handed individuals (eight women) between 19 and 34 years (mean age \pm SD: $23.8 \pm 3.4$ years) participated. The sample size was decided a priori based on similar experiments (Alsmith et al. 2017; Ferrè et al. 2013). The sample size was set in advance of testing and was also used as data-collection stopping rule. All participants were right-handed (Edinburgh Handedness Inventory, Oldfield, 1971) with normal or corrected-to-normal vision. Exclusion criteria included neurological, psychiatric or vestibular conditions, epilepsy or family history of epilepsy. The experimental protocol was approved by the Department of Psychological Sciences research ethics committee at Birkbeck, University of London. The study adhered to the ethical standards of the Declaration of Helsinki. Participants gave written informed consent to participate before inclusion in the experiment.

\section{Galvanic vestibular stimulation}

Bipolar Galvanic Vestibular Stimulation (GVS) was applied to deliver a boxcar pulse of $1 \mathrm{~mA}$ for $3 \mathrm{~s}$ using a commercial stimulator (Good Vibrations Engineering Ltd., Nobleton, Ontario, Canada). We have used event related, short duration and low-intensity boxcar GVS to avoid the lateralized cueing due to the strong sensation under the cathode during stimulation. Although we did not formally investigate the feelings induced by our stimulation, we know it was easily tolerable and not unpleasant for the participants. Some reported a slight feeling of rotation or 'dizziness'. Importantly, $1 \mathrm{~mA} \mathrm{GVS}$ is sufficient to provide effective vestibular stimulation, because it induces postural reflexes (Fitzpatrick and Day 2004). Carbon rubber electrodes (area $10 \mathrm{~cm}^{2}$ ) coated with electrode gel were placed binaurally over the mastoid processes and fixed in place with adhesive tape. The area of application was first cleaned and electrode gel was applied to reduce impedance. Both left anodal and right cathodal (Left GVS) and right anodal and left cathodal (Right GVS) configurations were used (Fig. 1B). Using this binaural bipolar configuration, GVS is known to increase the firing rate in vestibular afferents on the cathodal side and to decrease the firing rate on the anodal side (Goldberg et al. 1984). We also applied Sham stimulation using electrodes placed on the left and right side of the neck, about $5 \mathrm{~cm}$ below the
GVS electrodes (Lopez et al. 2010; Ferrè et al. 2013), with a left anodal and right cathodal configuration (Fig. 1B). Although the electrodes placed on the neck might induce different skin sensations and stimulate different nerves than the one placed behind the ears, this Sham stimulation can evoke similar tingling skin sensations to GVS, and so functioned as a control for non-specific effects.

\section{Experimental procedure}

Verbal and written instructions were given to participants at the beginning of the experiment. The experiment was administered in sitting position to reduce postural effects of GVS. The head was in a neutral posture, i.e., neither flexed nor tilted. Participants were asked to fixate the computer monitor with hands on a keyboard. Electrodes for GVS and Sham stimulation were placed at the beginning of the session and remained in place for the entire duration of the experiment. The electrodes and the polarity of stimulation were selected under computer control.

Stimuli for the Misalignment Paradigm were similar to our previous studies (Alsmith et al. 2017; Longo et al. 2020) and are shown in Fig. 1A. In each trial, the image of an avatar appeared. Stimuli were presented on a 24-inch monitor $(1024 \times 768$ pixels $)$ located approximately $40 \mathrm{~cm}$ in front of the participant under control of a custom LabVIEW (National Instruments Corporation, www.ni.com/ labview; Bitter et al. 2006) script. On each block of trials, the position of the avatar's torso was held constant with the torso (200 pixels in width, $10.63^{\circ}$ visual angle) centred on the monitor, oriented toward one of five compass directions (E, NE, N, NW, W), with the head rotated $45^{\circ}$ to either the right or left. This resulted in ten different orientations and each of the ten positions was presented once per vestibular stimulation condition. Presenting the body in different orientations ensured that participants were basing their judgments on a reference frame centred on the avatar depicted, rather than on their own body, visual field, or any other external cues. In each trial, a ball (21 pixels in diameter, $0.8^{\circ}$ ) appeared at nine angles evenly spaced between $-60^{\circ}$ and $+60^{\circ}$ degrees from the line midway between the head and torso. Participants were asked to make simple spatial judgments about the location of the ball with respect to the avatar depicted. For each angle, there were two distances of the ball from the person, Near $\left(6.39^{\circ}\right.$ from the centre of the head), and $\operatorname{Far}\left(19.17^{\circ}\right)$. This allowed to investigate a potential spatial gradient in the vestibular modulation of head and torso references. In the Misalignment Paradigm, the most informative judgments are the ones in which the ball appears at the three centre angles $\left(0^{\circ}, 15^{\circ},-15^{\circ}\right)$. In these trials, the ball could be judged to the avatar's right or to the avatar's left in function of the references used by the participant. Please 
A

The Misalignment Paradigm

B Left GVS
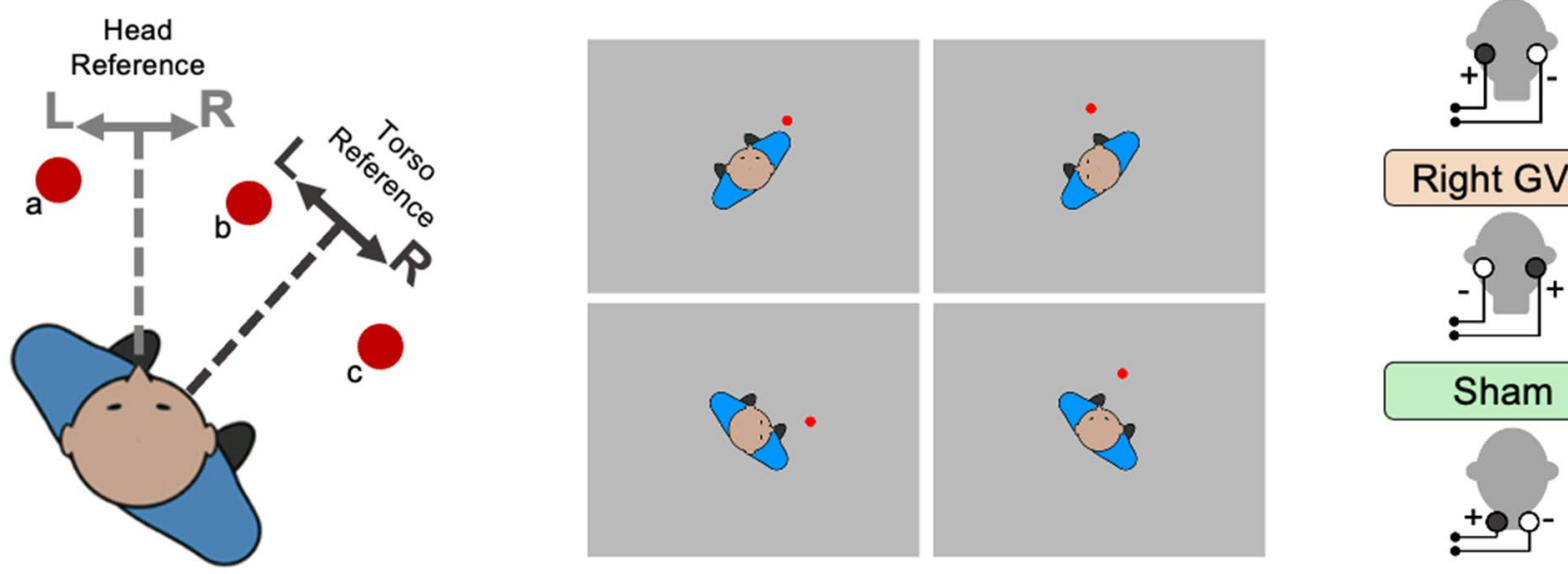

Right GVS

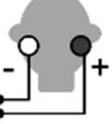

Sham

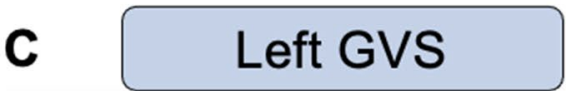

Right GVS
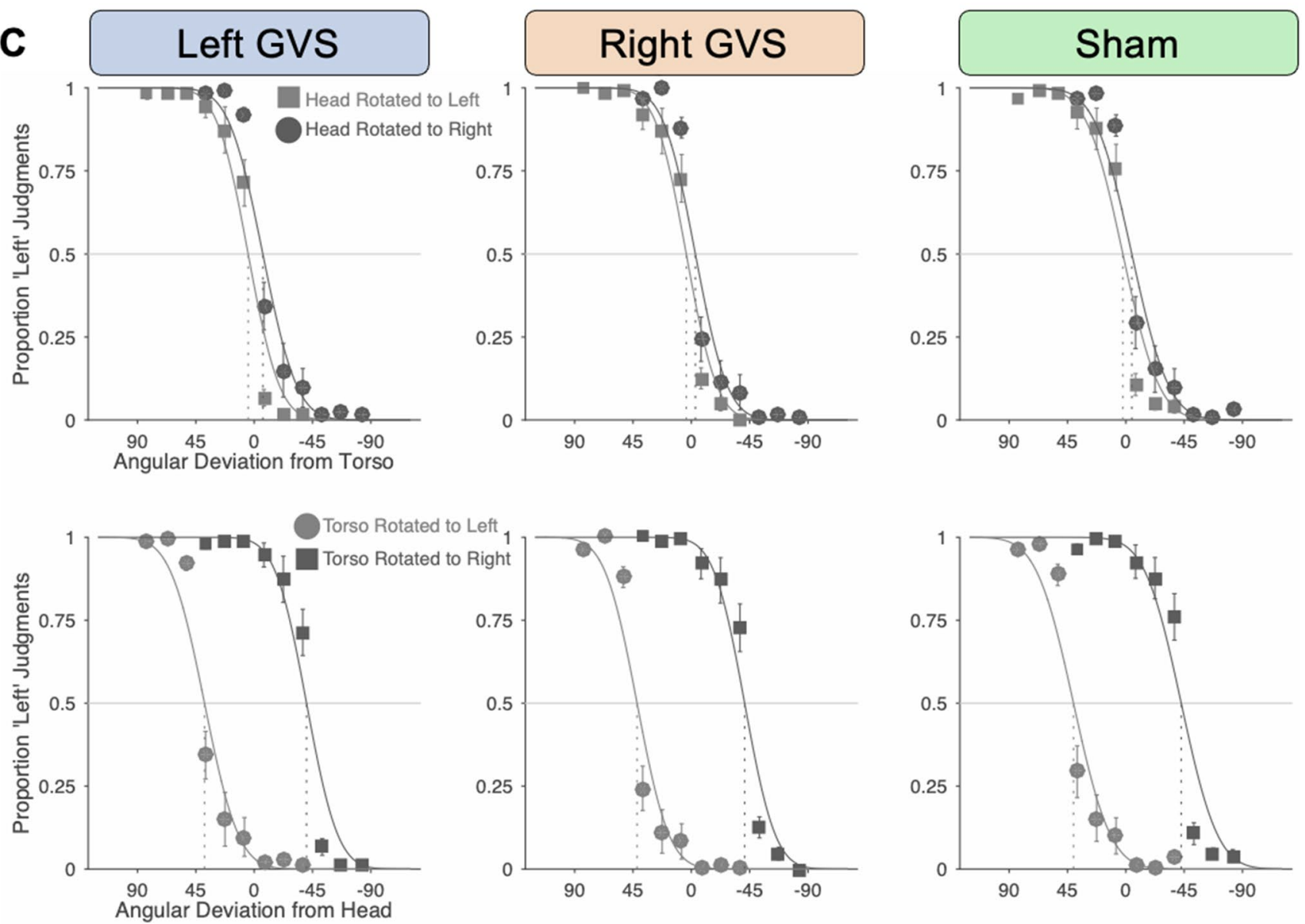

Fig. 1 Experimental set up and results. A The Misalignment Paradigm (adapted from Alsmith et al. 2017). The locations of balls a and c are clearly at the left and right of the person, while the location of ball $b$ is critical; if the torso is the origin of the egocentric reference frame, ball c is to the person's left; if the head is the origin, it is to their right. B Examples of visual stimuli used in the Misalignment Paradigm. C GVS configuration. Both left anodal and right cathodal (Left GVS) and right anodal and left cathodal (Right GVS) configura- tions were used. A Sham stimulation with the electrodes placed on the left and right side of the neck was adopted to control for non-specific effects. D Proportion of 'Left' judgements as function of angular deviation from the torso and head. The weight given to the torso was overall greater than that given to the head. Critically, artificial vestibular stimulation modulated these weights; Left GVS increased the weighting given to the head compared to Right GVS or Sham stimulation Alsmith et al. (2017) 
consider Fig. 1A, ball b. If the torso is used as origin of the egocentric reference frame, the ball is to the avatar's left; if the head is the origin, it is to their right. To maximize the number of most informative judgments, within each distance the three centre angles $\left(0^{\circ}, 15^{\circ},-15^{\circ}\right)$ were each presented three times, the next three most extreme on each side $\left(30^{\circ}, 45^{\circ}, 60^{\circ},-30^{\circ},-45^{\circ},-60^{\circ}\right)$ were each presented twice, and the most extreme angles $\left(75^{\circ}\right.$, $\left.90^{\circ},-75^{\circ},-90\right)$ were each presented once for each vestibular stimulation condition (Left-GVS, Right-GVS and Sham stimulation). A total of 75 trials was presented. Left GVS, Right GVS or Sham stimulation was applied for $3 \mathrm{~s}$ in each trial. The stimulation was delivered after $2 \mathrm{~s}$ from the beginning of the trial, then after $1 \mathrm{~s}$ the ball appeared for $2 \mathrm{~s}$. After that, the ball disappeared and GVS was also turned off. Participants were instructed to "judge whether the ball is to the person's left or to their right". They made responses by pressing the ' $\mathrm{q}$ ' key on the keyboard with the left index finger if they judged the ball as being to the person's left and the 'p' key with their right index finger if they judged it as being to the person's right. Participants were instructed to make the judgments within the $2 \mathrm{~s}$ time window in which the stimulation was on and the ball displayed on the screen. To avoid aftereffects of GVS on the subsequent trial, there was an inter-trial-interval of $3 \mathrm{~s}$. The body remained on the screen during the interval. GVS conditions were applied in separate blocks and the order of GVS conditions was counterbalanced. The experiment lasted about 1 hour.

\section{Analysis}

The analysis was similar to that used in our previous study (Alsmith et al. 2017; Longo et al. 2020). Best-fitting cumulative Gaussian functions were fit using maximum-likelihood estimation for each participant in each condition using the Palamedes toolbox for MATLAB (Mathworks, Natick, MA, Prins and Kingdom (2009), available online at: http://www. palamedestoolbox.org). To isolate contributions of the head, we analysed responses as a function of angular deviation of the ball from an axis aligned with the torso, comparing the conditions in which the head was rotated to the right vs. to the left. Conversely, to isolate contributions of the torso, we analysed responses as a function of angular deviation of the ball from an axis aligned with the head, comparing the conditions in which the torso was rotated to the right vs. to the left. For each psychometric function, the Point of Subjective Equality (PSE) was estimated. We quantified the contribution of the head and of the torso by calculating the PSE Shift for each body part, defined as the difference in PSE between the conditions in which the relevant part was rotated to the left and to the right. Because the total PSE
Shift for the head and for the torso must add to $90^{\circ}$, we also calculated the proportionate weighting given to each of the two body parts by dividing the PSE Shift by $90^{\circ}$.

\section{Results}

Psychometric functions showed an excellent fit to the data, with a mean $R^{2}$ of 0.960 (range $0.683-1$ ). As no effects of distance were found, the near and far distances were collapsed in Fig. 1C and subsequent analyses. Individual subjects and Mean PSE Shift data as function of vestibular stimulation conditions (Left GVS, Right GVS, Sham) are reported in Fig. 2.

There were clear contributions of the torso in the Sham stimulation (Mean PSE Shift $79.5^{\circ}$, Mean weighting 0.884), $t(14)=12.30, p<0.0001, d_{z}=3.18$, Left GVS (Mean PSE Shift $74.9^{\circ}$, Mean weighting 0.832), $t(14)=11.84$, $p<0.0001, d_{z}=3.06$, and Right GVS (Mean PSE Shift $80.3^{\circ}$, Mean weighting 0.893$), t(14)=13.74, p<0.0001$, $d_{z}=3.55$, conditions. Contributions of the head were less clear than in our previous study (Alsmith et al. 2017). While there was a significant contribution of the head in the Left GVS condition (Mean PSE Shift $15.1^{\circ}$, Mean weighting

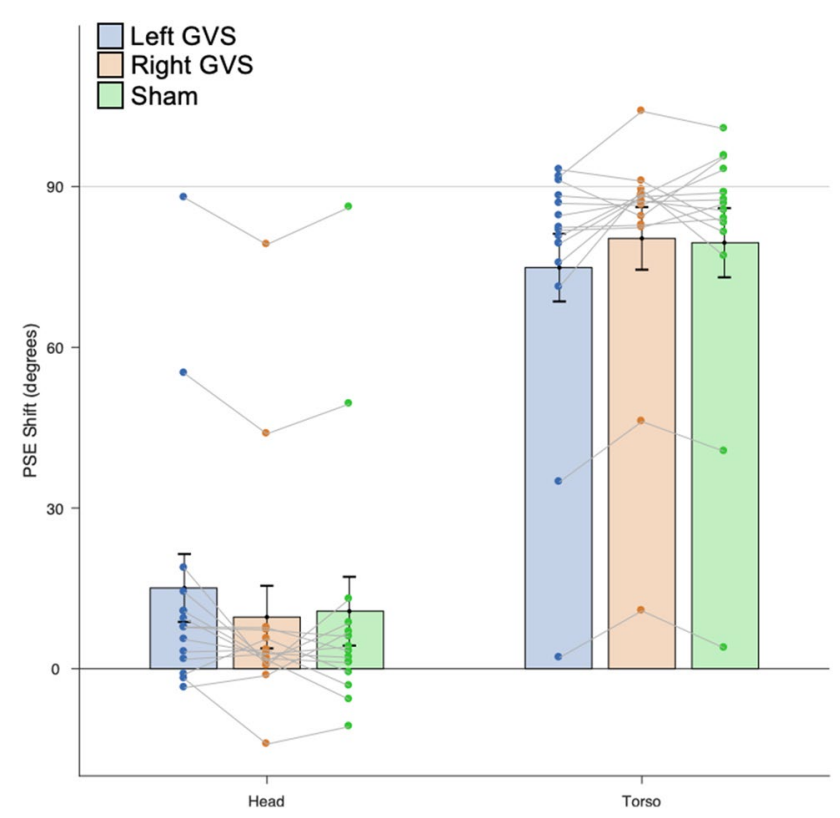

Fig. 2 PSE shift as function of vestibular stimulation conditions. The contribution of the head and of the torso was quantified by calculating the PSE Shift for each body part, defined as the difference in PSE between the conditions in which the relevant part was rotated to the left and to the right. Because the total PSE Shift for the head and for the torso must add to $90^{\circ}$, we also calculated the proportionate weighting given to each of the two body parts by dividing the PSE Shift by $90^{\circ}$. PSE shifts in each experimental conditions are presented as single subject data and average across participants. Bars indicate standard error 
$0.168), t(14)=2.38, p=0.03, d=0.61$, this did not reach significance in the Sham Stimulation (Mean PSE Shift: $10.7^{\circ}$, Mean weighting: 0.119$), t(14)=1.67, p=0.12, d=0.43$, or Right GVS (Mean PSE Shift 9.6 ${ }^{\circ}$, Mean weighting 0.107), $t(14)=1.65, p=0.12, d=0.43$, conditions.

An ANOVA on PSE Shifts revealed a main effect of Body Part (Head, Torso), $F(1,14)=29.35$, MSE $=3382.90$, $p<0.0001, \eta_{\mathrm{p}}{ }^{2}=0.68$. Critically, this effect was modulated by a significant interaction between body part and stimulation condition, $F(2,28)=5.78, \mathrm{MSE}=43.84, p=0.008$, $\eta_{\mathrm{p}}{ }^{2}=0.29$. There were no other significant main effects or interaction $(p>0.05)$. Follow-up testing using the Bonferroni Holm procedure showed that Left GVS increased the weight given to the head compared to both Right GVS, $t(14)=3.03, p=0.009, d_{z}=0.78$, and Sham stimulation, $t(14)=2.48, p=0.02, d_{z}=0.64$. There was no differences between Right GVS and Sham stimulation $t(14)=0.71$, $p=0.49$.

\section{Discussion}

The vestibular system provides a head-related signal, constantly detecting head acceleration and orientation in the three-dimensional space (Lackner and DiZio 2005). Our data showed that the weight given to the head in determining an object's location was increased during left anodal and right cathodal GVS, compared to the opposite GVS polarity and Sham stimulation. That is, the polarity of GVS, which preferentially activates vestibular areas in distinct cerebral hemispheres, had differential effects on the weighting of head and torso in egocentric spatial judgements.

Previous research investigating the contribution of different body parts to egocentric spatial judgements showed that both the head and the torso contribute to the determination of egocentric representation, with slightly greater reliance on the torso (Alsmith et al. 2017). In our knowledge, no studies focused on whether the contribution of these body parts to egocentric spatial judgments is a stored representation reflecting semantic knowledge about the body morphology, or a dynamic, online updated sensory representation, perhaps reflecting the saliency of afferent sensory signals. Our results showed a change in the weight given to the head and torso when artificial vestibular stimulation was delivered to enhance vestibular processing. This suggests that the saliency of afferent sensory signals plays a role in the weight given to different body parts while making egocentric spatial judgements.

GVS polarity-dependent differences in postural, sensorimotor and cognitive functions have been demonstrated both in healthy volunteers and in brain damaged patients. This might arise if one polarity of GVS has stronger effects in the brain, perhaps reflecting a cerebral dominance for vestibular processing. Accordingly, neuroimaging studies have identified an asymmetry in the cortical vestibular system, suggesting that the cortical vestibular network is primarily located in the non-dominant right hemisphere in right handed participants (Dieterich et al. 2003; Bense et al. 2001; Suzuki et al. 2001; Janzen et al. 2008). Therefore, the polarity-specific influence of left anodal and right cathodal GVS on the head weight in the Misalignment Paradigm may be related to modulations of mechanisms encoding egocentric representations in the right hemisphere. However, the mechanism that links GVS polarity effects to cortical dominance remains still unclear. In particular, one might imagine that the dominant right hemisphere vestibular projections could be activated by both left anodal and right cathodal GVS and right anodal and left cathodal GVS (Eickhoff et al. 2006a, b), yet we found effects only of left anodal and right cathodal GVS. However, fMRI studies identified a relatively strong activation of the right hemisphere during left anodal and right cathodal GVS compared to the opposite polarity (Fink et al. 2003). Thus, right anodal and left cathodal GVS may have simply been not strong enough to modulate the egocentric frame of reference.

Clinical reports have shown that a unilateral lesion to the vestibular peripheral organ yields to a tonic imbalance in vestibular processing which may contribute to postural, balance and gait problems, including head and trunk tilt deviation to the lesioned side (Borel et al. 2008). Alterations in the representation of body orientations have also been described after unilateral vestibular loss (Saj et al. 2013). Interestingly, only patients with left vestibular loss, a vestibular asymmetry which is consistent to that created by left anodal and right cathodal GVS, showed more severe biases in the representation of self-orientation in space. Taken together these results converge with a right hemispheric dominance for space representation, as well as a right hemispheric dominance of cortical vestibular projections (Bottini et al. 1994; Dieterich et al. 2003).

Previous studies have investigated the relation between body parts orientation and spatial attention. Grubb and Reed (2002) observed a pseudoneglect bias in a covert attention task by leftward rotation of the torso. Hasselbach-Heitzeg and Reuter-Lorenz (2002) also found that rightward rotation reduced response times for targets on the right. Potentially, GVS could have affected egocentric spatial judgements indirectly through attentional or arousal mechanisms, rather than through any direct effects on egocentric representation. Left anodal and right cathodal GVS produces shift in spatial attention toward the left space, whereas right anodal and left cathodal GVS induces an attentional bias toward the right space (Ferrè et al. 2013). In Left GVS trials, the attention of participants might have been shifted toward the left hemispace. However, this general shift could not explain the 
clear interaction we found between GVS polarity and head vs torso judgements. This interaction was due to the head references only: there was no difference between Left GVS and Right GVS in the torso judgments. Thus, an explanation based on shifts of spatial attention cannot readily account for our results. Accordingly, Rorden et al. (2001) also found that inducing illusions of torso rotation did not produce effects in attentional orientation.

The Misalignment Paradigm suggests that egocentric spatial judgments involve the use of reference frames centred both on the head and torso, with differences between people in the use of these body parts as anchor (Alsmith et al. 2017; Longo et al. 2020). Although the factors that may drive individual differences in the use of these body parts or in the use of a weighted combination of head and torso are not yet entirely clear, we have recently shown an high stability across time (Longo et al. 2020). Participants re-tested on the Misalignment Paradigm several months after the original test showed a strong correlation between the two testing sessions in their use of head vs. torso references. However, further research might focus specifically on the drivers of these differences.

It is important to note that the Misalignment Paradigm measured egocentric spatial judgments using a third-person perspective taking task in which participants are explicitly being asked to make judgments of spatial position with respect to a seen avatar. Thus, the effects of vestibular stimulation on head and torso references do not involve location of targets directly from the participant's first-person perspective. Recent studies have demonstrated vestibular modulation of third-person perspective taking both in healthy participants (Deroualle et al. 2015) and vestibular patients (Deroualle et al. 2019). Interestingly, only patients with left vestibular loss presented altered third-person perspective taking compared to controls. No impairment was observed in first-person perspective taking and the 3D objects mental imagery abilities (Deroualle et al. 2019). These results are in agreement with the findings of the present study, supporting the importance of a right cortical vestibular network for the cognitive representation of the body in space.

In conclusion, our study highlights vestibular contributions to egocentric representation. Egocentric spatial judgments rely on a weighted combination of reference frames centred on at least two different parts of the body: the head and torso. We have shown that afferent vestibular signals modulates the relative weightings of head and torso, showing that vestibular information contributes to computation of egocentric representations.

Acknowledgements This research was supported by grants from the Volkswagen Stiftung to AJTA and MRL and from the European Research Council (ERC-2013-StG-336050) to MRL. AJTA was additionally supported by ERC-2017-STG (757698). ERF and PH were supported by European Union Seventh Framework Programme (EU
FP7) project VERE WP1. PH was additionally supported by a Professorial Fellowship from the ESRC and by ERC Advanced Grant HUMVOL.

Data availability The datasets generated during and/or analysed during the current study are available from the corresponding author on reasonable request.

\section{Declarations}

Conflict of interest The authors declared that they had no conflicts of interest with respect to their authorship or the publication of this article.

Open Access This article is licensed under a Creative Commons Attribution 4.0 International License, which permits use, sharing, adaptation, distribution and reproduction in any medium or format, as long as you give appropriate credit to the original author(s) and the source, provide a link to the Creative Commons licence, and indicate if changes were made. The images or other third party material in this article are included in the article's Creative Commons licence, unless indicated otherwise in a credit line to the material. If material is not included in the article's Creative Commons licence and your intended use is not permitted by statutory regulation or exceeds the permitted use, you will need to obtain permission directly from the copyright holder. To view a copy of this licence, visit http://creativecommons.org/licenses/by/4.0/.

\section{References}

Alsmith AJ, Longo MR (2014) Where exactly am I? Self-location judgements distribute between head and torso. Conscious Cogn 24:70-74

Alsmith AJ, Ferrè ER, Longo MR (2017) Dissociating contributions of head and torso to spatial reference frames: the misalignment paradigm. Conscious Cogn 53:105-114

Avillac M, Denève S, Olivier E, Pouget A, Duhamel J-R (2005) Reference frames for representing visual and tactile locations in parietal cortex. Nat Neurosci 8:941-949

Bense S, Stephan T, Yousry TA, Brandt T, Dieterich M (2001) Multisensory cortical signal increases and decreases during vestibular galvanic stimulation (fMRI). J Neurophysiol 85:886-899

Bermúdez JL (1998) The paradox of self-consciousness. MIT Press, Cambridge, MA

Bermúdez JL (2005) The phenomenology of bodily awareness. Phenomenology and philosophy of mind. Oxford University Press, pp 295-322

Bitter R, Mohiuddin T, Nawrocki M (2006) LabVIEW: advanced programming techniques. Crc Press

Blouin J, Gauthier GM, Vercher JL, Cole J (1996) The relative contribution of retinal and extraretinal signals in determining the accuracy of reaching movements in normal subjects and a deafferented patient. Exp Brain Res 109(1):148-153

Blouin J, Labrousse L, Simoneau M, Vercher JL, Gauthier GM (1998) Updating visual space during passive and voluntary head-in-space movements. Exp Brain Res 122(1):93-100

Bonnier P (1905) L'aschématie. Rev Neurol (paris) 13:605-609

Borel L, Lopez C, Péruch P, Lacour M (2008) Vestibular syndrome: a change in internal spatial representation. Neurophysiol Clinique/ clin Neurophysiol 38(6):375-389

Bottini G, Sterzi R, Paulesu E, Vallar G, Cappa SF, Erminio F, Frackowiak RS (1994) Identification of the central vestibular 
projections in man: a positron emission tomography activation study. Exp Brain Res 99(1):164-169

Clement G, Fraysse MJ, Deguine O (2009) Mental representation of space in vestibular patients with otolithic or rotatory vertigo. NeuroReport 20(5):457-461

Deecke L, Schwarz DWF, Fredrickson JM (1977) Vestibular responses in the rhesus monkey ventroposterior thalamus. II. Vestibuloproprioceptive convergence at thalamic neurons. Exp Brain Res 30(2-3):219-232

Deroualle D, Lopez C (2014) Towards a vestibular contribution to social cognition. Front Integr Neurosci 8:16

Deroualle D, Borel L, Devèze A, Lopez C (2015) Changing perspective: the role of vestibular signals. Neuropsychologia 79:175-185

Deroualle D, Borel L, Tanguy B, Bernard-Demanze L, Devèze A, Montava M, Lopez C (2019) Unilateral vestibular deafferentation impairs embodied spatial cognition. J Neurol 266(1):149-159

Dieterich M, Bense S, Lutz S, Drzezga A, Stephan T, Bartenstein P, Brandt T (2003) Dominance for vestibular cortical function in the non-dominant hemisphere. Cereb Cortex 13:994-1007

Eickhoff SB, Weiss PH, Amunts K, Fink GR, Zilles K (2006a) Identifying human parieto-insular vestibular cortex using fMRI and cytoarchitectonic mapping. Hum Brain Mapp 27:611-621

Eickhoff SB, Weiss PH, Amunts K, Fink GR, Zilles K (2006b) Identifying human parieto-insular vestibular cortex using fMRI and cytoarchitectonic mapping. Hum Brain Mapp 27(7):611-621

Ferrè ER, Longo MR, Fiori F, Haggard P (2013) Vestibular modulation of spatial perception. Front Hum Neurosci 7:660

Fink GR, Marshall JC, Weiss PH, Stephan T, Grefkes C, Shah NJ, Dieterich M (2003) Performing allocentric visuospatial judgments with induced distortion of the egocentric reference frame: an fMRI study with clinical implications. Neuroimage 20:1505-1517

Fitzpatrick RC, Day BL (2004) Probing the human vestibular system with galvanic stimulation. J Appl Physiol 96:2301-2316

Goldberg J, Smith C, Fernandez C (1984) Relation between discharge regularity and responses to externally applied galvanic currents in vestibular nerve afferents of the squirrel monkey. J Neurophysiol 51:1236-1256

Grubb JD, Reed CL (2002) Trunk orientation induces neglect-like lateral biases in covert attention. Psychol Sci 13(6):553-556

Hasselbach-Heitzeg MM, Reuter-Lorenz PA (2002) Egocentric bodycentered coordinates modulate visuomotor performance. Neuropsychologia 40(11):1822-1833

Janzen J, Schlindwein P, Bense S, Bauermann T, Vucurevic G, Stoeter P, Dieterich M (2008) Neural correlates of hemispheric dominance and ipsilaterality within the vestibularsystem. Neuroimage 42:1508-1518

Jeannerod M (1988) The neural and behavioural organization of goaldirected movements. Clarendon Press, Oxford

Jeannerod M, Biguer B (1987) The directional coding of reaching movements. A visuomotor conception of spatial neglect. Adv Psychol 45:87-113 (North-Holland)

Karnath HO, Schenkel P, Fischer B (1991) Trunk orientation as the determining factor of the 'contralateral' deficit in the neglect syndrome and as the physical anchor of the internal representation of body orientation in space. Brain 114:1997-2014
Knox JJ, Coppieters MW, Hodges PW (2006) Do you know where your arm is if you think your head has moved? Exp Brain Res 173(1):94-101

Lackner JR, DiZio P (2005) Vestibular, proprioceptive, and haptic contributions to spatial orientation. Annu Rev Psychol 56:115-147

Lenggenhager B, Lopez C (2015) Vestibular contributions to the sense of body, self, and others. In: Metzinger T, Windt JM (eds) Open MIND: 23(T). MIND Group, Frankfurt am Main

Lhermitte J (1952) L'image corporelle en neurologie. Schweiz Arch Neurol Psychiatr 69:214-236

Longo MR, Rajapakse SS, Alsmith AJ, Ferrè ER (2020) Shared contributions of the head and torso to spatial reference frames across spatial judgments. Cognition 204:104349

Lopez C, Lenggenhager B, Blanke O (2010) How vestibular stimulation interacts with illusory hand ownership. Conscious Cogn 19:33-47

Lopez C, Blanke O, Mast FW (2012) The human vestibular cortex revealed by coordinate-based activation likelihood estimation meta-analysis. Neuroscience 212:159-179

Pavlidou A, Ferrè ER, Lopez C (2018) Vestibular stimulation makes people more egocentric. Cortex 101:302-305

Peacocke C (1992) A study of concepts. MIT Press, Cambridge, MA

Prins N, Kingdom FAA (2009) Palamedes: Matlab routines for analyzing psychophysical data

Rorden C, Karnath HO, Driver J (2001) Do neck-proprioceptive and caloric-vestibular stimulation influence covert visual attention in normals, as they influence visual neglect? Neuropsychologia 39(4):364-375

Saj A, Honoré J, Bernard-Demanze L, Devèze A, Magnan J, Borel L (2013) Where is straight ahead to a patient with unilateral vestibular loss? Cortex 49(5):1219-1228

Schilder P (1935) The image and appearance of the human body. International Univ. Press, New York

Schwarz DWF, Deecke L, Fredrickson JM (1973) Cortical projection of group I muscle afferents to areas 2, 3a, and the vestibular field in the rhesus monkey. Exp Brain Res 17(5):516-526

Serino A, Noel JP, Galli G, Canzoneri E, Marmaroli P, Lissek H, Blanke O (2015) Body part-centered and full body-centered peripersonal space representations. Sci Rep 5:18603

Sherrington C (1907) On the proprio-ceptive system, especially in its reflex aspect. Brain 29:467-482

Suzuki M, Kitano H, Ito R, Kitanishi T, Yazawa Y, Ogawa T, Shiino A, Kitajima K (2001) Cortical and subcortical vestibular response to caloric stimulation detected by functional magnetic resonance imaging. Cogitive Brain Research 12:441-449

Zu Eulenburg P, Caspers S, Roski C, Eickhoff SB (2012) Meta-analytical definition and functional connectivity of the human vestibularcortex. Neuroimage 60(1):162-169

Publisher's Note Springer Nature remains neutral with regard to jurisdictional claims in published maps and institutional affiliations. 